\section{Frequency response characteristics for sinusoidal movement in the fovea and periphery*}

\author{
C. WILLIAM TYLER and JEAN TORRES \\ Northeastern University, Boston, Massachusetts 02115
}

Threshold sensitivity was measured for sinusoidal movement of bright 1-deg lines against a dark background as a function of oscillation frequency and retinal location. Sensitivity was greatest in the fovea and at a frequency of $1-2 \mathrm{~Hz}$. Peripheral sensitivity was more narrowly tuned than foveal sensitivity. The presence of a stationary reference line affected mainly the foveal sensitivity. The results are interpreted as evidence for both position- and velocity-sensitive mechanisms in the movement detection system.

The techniques of analysis of linear systems were first applied to the study of visual function by de Lange (1952, $1958 \mathrm{a}, \mathrm{b})$ in his experiments on threshold flicker. The systems analysis approach, often involving the use of sinusoidal stimuli, has found wide acceptance among vision researchers. By measuring threshold amplitudes; sensitivity can be plotted as a function of frequency of the sinusoid, on log-log coordinates. This type of plot is called a frequency response function $^{1}$ (FRF). Its use greatly facilitates understanding of the processes underlying the system response.

FRFs for sine-wave flicker have been obtained as a function of variables such as retinal adaptation level (Kelly, 1961), field size (van der Tweel, 1960), chromatic stimulation (de Lange, 1958a, b; Kelly, 1962), and spatial pattern (Kelly, 1969a; Levinson, 1964). Keesey (1970) has studied the effects of target size, luminance, surround, and retinal-image movement on sensitivity to sinusoidal flicker. Kelly (1969a) has derived the temporal properties of a lateral interaction mechanism. The systems analysis approach has also been successfully applied to aspects of temporal visual function other than flicker. Schickman (1970), for example, has used low-frequency sinusoidally modulated light as a masking stimulus for brief light flashes. Wavelength-modulated light has been used in a systems analysis approach to color vision (Regan \& Tyler, 1971a, b).

In the field of movement perception, investigations of both apparent movement for square-wave modulated displacement (Tyler, 1972a) and stereoscopic depth movement for sinusoidal line displacement (Tyler, 1971) have revealed important ixiumation concerning the respective

*Supported by the Foundations Fund for Research in Psychiatry, Grant 70-481. processing systems by the use of frequency response analysis. Clemmesen (1944) has used sinusoidally modulated displacement in his work on the perception of moving targets in the fovea and periphery. However, complete FRFs for movement sensitivity have not been reported in any of the literature surveyed.

The experiments described in this paper serve the twofold purpose of measuring the FRF of the human observer to sinusoidally moving targets in the fovea and periphery, and of deducing the nature of the visual system in determining the form of the FRF. Although few investigators have worked with sinusoidal motion, data are available on the variation in sensitivity to linear and rotary motion as a function of retinal position. McColgin (1961) found that rate sensitivity for these two types of motion varied in a similar manner, the threshold velocity increasing linearly with eccentricity at a somewhat greater rate along the vertical than

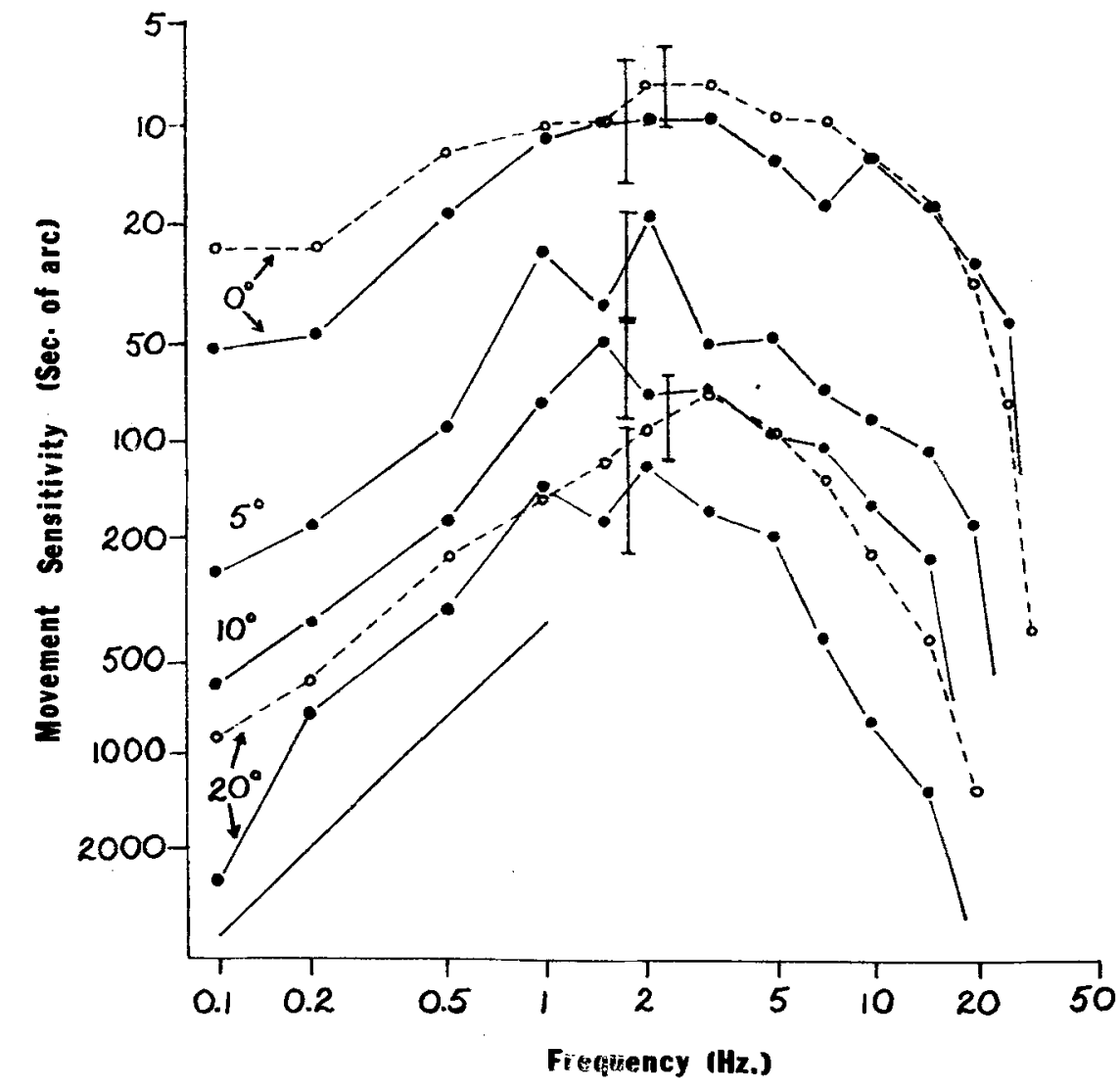

Fig. 1. Threshold sensitivity in seconds of arc to sinusoidal movement with a stationary reference, plotted as the reciprocal of threshold amplitude, as a function of oscillation frequency. Retinal eccentricity is the parameter of the four curves. Eccentricities used were 0, 5, 10, and 20 deg, reading downwards on the graph. Ss were J.T. (filled circles) and C.W.T. (open circles). Vertical bars represent one standard deviation on each side of the mean. An indication is given of the slope of sensitivity above the highest frequency for visible movement. These lines are the shallowest slope possible, since movement was invisible at the next frequency. Note decreasing sensitivity and change in form of curves with increasing eccentricity. The oblique straight line represents slope of unity approximated by peripheral curves at low frequencies. 


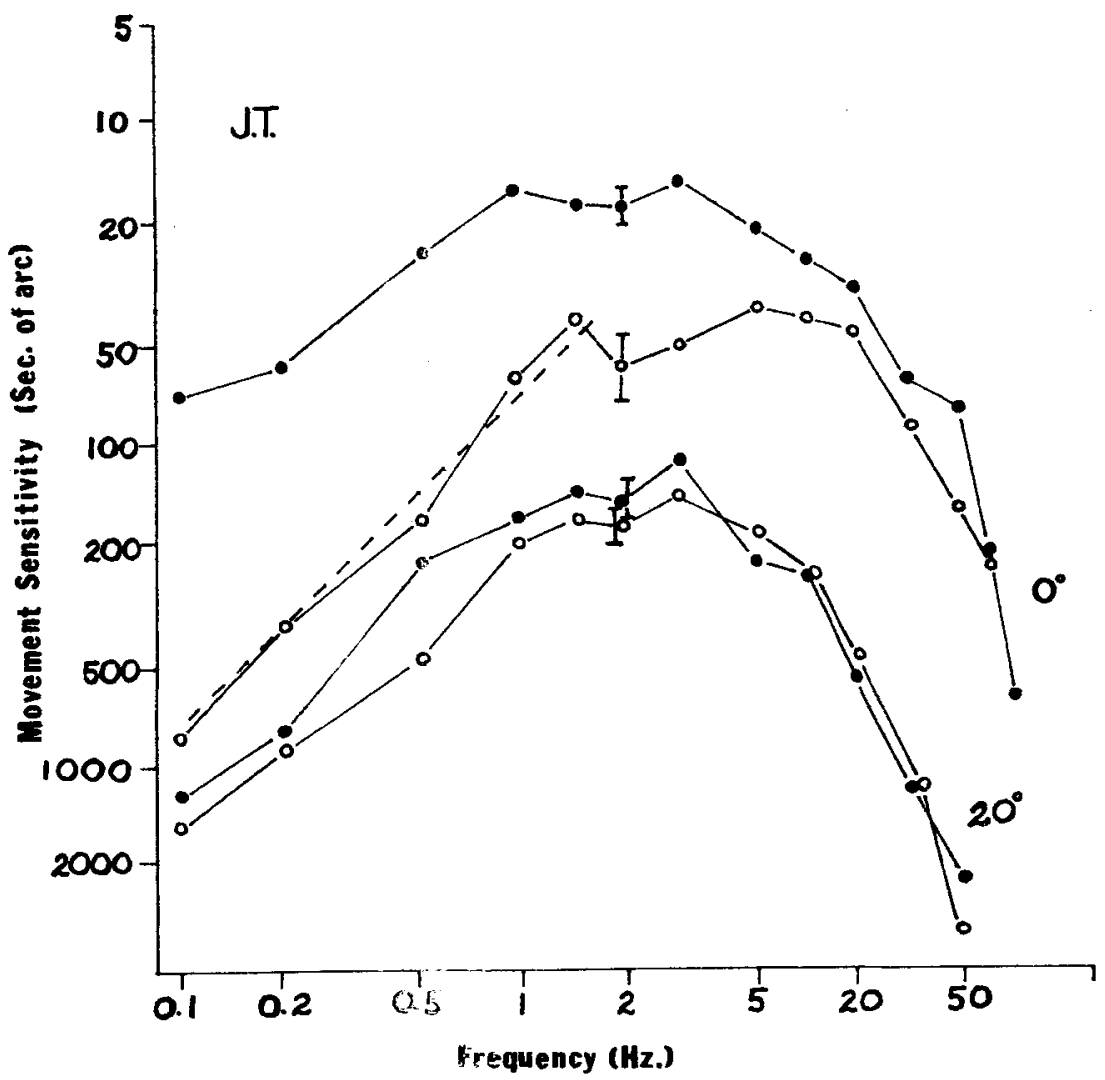

Fig. 2. Threshold sensitivity in seconds of are to sinusoidal movement with reference (filled circles) and without reference (open circles). S J.T.'s upper curves show 0 deg eccentricity, lower curves 20 deg eccentricity. Bars represent one standard deviation on each side of mean. The dashed line shows a slope of unity. Note differential effects of removal of reference at 0 and $20 \mathrm{deg}$ eccentricity.

along the horizontal meridian. However, he does not give data that are detailed enough to permit comparison between foveal and 20-deg peripheral sensitivities, as will be done in the present experiment.

\section{EXPERIMENT I}

Apparatus and Method

A fast-phosphor dual-beam oscilloscope was used for presentation of two vertical lines, at 10-fL luminance against a dark surround. The frequency response of the phosphor was $200 \mathrm{~Hz} \pm 3 \mathrm{~dB}$. A very dim background glow from the CRT was perceptible. One line was held steady, while the second line could be sinusoidally displaced in the horizontal direction by means of a variable frequency function generator. Any effect of position cues from the CRT glow was certainly negligible in relation to the bright reference line. At a fixation distance of $3 \mathrm{~m}$, both lines were $1 \mathrm{deg}$ high and approximately $1 \mathrm{~min}$ wide. They were viewed binocularly with natural pupil, either in central vision or with fixation on a bright vertical bar target located at 5 ,
10 , or 20 deg subtense from the center of the stimulus. Dark adaptation was not necessary since the stimuli were of photopic luminance. Thresholds were measured by the method of adjustment, with an equal number of ascending and descending measurements being taken at each of a range of frequencies from 0.1 to $30 \mathrm{~Hz}$. The $S$ adjusted a linear potentiometer to determine the just-stationary threshold. The E altered the gain of the oscilloscope so that the potentiometer setting was always near the optimal position. For foveal viewing, the reference line was placed $10 \mathrm{~min}$ away from the target, while for peripheral viewing it was located at a distance of $30 \mathrm{~min}$. Pilot experiments on the effects of angular distance between reference line and target indicate that the distance of the reference line has very little effect on the movement sensitivity within wide limits. For example, varying line separation in foveal viewing from 10 to $30 \mathrm{~min}$ affected the thresholds by only a factor of 1.5 at both 0.5 and $5 \mathrm{~Hz}$. Due to difficulty with the apparatus, measurements were taken first for the frequencies of $0.5 \mathrm{~Hz}$ and higher for S J.T. At a later date, additional readings were taken for the lower frequencies, between 0.1 and $1.0 \mathrm{~Hz}$, the later data being calibrated with the earlier measurements to obtain the final curve. A total of eight measurements were taken for the frequencies below $1.0 \mathrm{~Hz}$. Data were also obtained for a second $S$ (C.W.T.) in the fovea and $20 \mathrm{deg}$ in the periphery, two measurements being taken at each frequency. Both Ss wore full refractive correction during experiments.

\section{Results and Discussion}

The results of Experiment I are shown in Fig. 1 for J.T. (full lines) and C.W.T. (dashed lines). The form of the FRF is similar for the two Ss, both in the fovea and at $20 \mathrm{deg}$ in the periphery. The most notable result is that sensitivity to sinusoidal movement is highest in the fovea at all frequencies. Sensitivity decreases as distance of the retinal image from the fovea increases. Earlier research by Clemmesen (1944) shows that at 2 or $3 \mathrm{~Hz}$ oscillation frequency, sensitivity in the fovea is greater than at $5 \mathrm{deg}$ eccentricity by a factor of 3. A similar difference is evident in Fig. 1 . The apparent peaks in sensitivity in the peripheral curves between 0.7 and $7 \mathrm{~Hz}$ are probably not repeatable. They are not consistent from one curve to the next and do not reach significance at $p=.01$ on a two-tailed $t$ test.

Clemmesen (1944) does not report any threshold measurements for frequencies other than 2.0 and $3.0 \mathrm{~Hz}$, but he states that between 1.1 and $5.0 \mathrm{~Hz}$, sensitivity was not affected by frequency. The present data for all retinal positions are consistent with these findings, as the differences across the range of 1 to $5 \mathrm{~Hz}$ are within one standard deviation of the sensitivity at $1.5 \mathrm{~Hz}$. (A single exception to this statement is shown by the 5-deg measurement at $2 \mathrm{~Hz}$, but two exceptions would be expected by chance alone, since a difference of one standard deviation represents a confidence level of $p=.15$, or one exception in every seven cases.) Between 0.2 and $5 \mathrm{~Hz}$, the decrease in movement sensitivity with increasing retinal angle from the fovea is approximately uniform across frequencies for S J.T. (full lines). Thus, the largest change, about $0.5 \mathrm{log}$ unit, occurs between 0 and $5 \mathrm{deg}$ of retinal angle. The differences from 5 to $10 \mathrm{deg}$ and from 10 to $20 \mathrm{deg}$ are similar at about $0.3 \mathrm{log}$ unit each.

The data of S C.W.T. (dashed lines) are in accord with these differences in showing a total decrease in sensitivity of close to $1.0 \mathrm{log}$ unit between 0 and 
20 deg, which is close to the total decrease of $1.1 \mathrm{log}$ units for $\mathbf{S}$ J.T. At frequencies greater than about $5 \mathbf{H z}$, it is no longer the case that sensitivity is independent of frequency or that the decrease in sensitivity with retinal angle is uniform acroes frequencies. The foveal sensitivity remains relatively high, while peripheral sensitivity shows a relative decrease as frequency increases. Thus, by $15 \mathrm{~Hz}$, sensitivity at 20 deg is reduced by $1.3 \mathrm{log}$ units for C.W.T. and by $1.85 \mathrm{log}$ units for J.T. The peripheral sensitivity is thus more sharply tuned than foveal sensitivity in the high-frequency region.

For both $\mathrm{Ss}$, the peripheral data below $1 \mathrm{~Hz}$ conform to a slope of unity (shown as the straight line in Fig. 1) within the limits of experimental error. The foveal sensitivity appears to level off somewhat at very low frequencies and is thus more broadly tuned than peripheral sensitivity at both high and low frequencies. This observation suggested that foveal and peripheral low-frequency movement detection might involve different types of processing systems. This possibility was investigated in a second experiment in which the effect of the removal of the reference line in the movement frequency response was examined.

\section{EXPERIMENT II}

Apparatus and Method

The conditions were the same as for Experiment I, except that the stimulus was presented without the reference line. One ascending and one descending series of measurements were taken, for the fovea and 20-deg peripheral fixation only, with the same Ss.

Arguments are presented below that the results do not appear to be contaminated by eye movements. As an empirical check, a subsidiary experiment was designed. The problem is to provide a fixation stimulus that does not simultaneously act as a position reference. Since eye tracking ability is negligible above $3 \mathrm{~Hz}$ (St. Cyr \& Fender, 1969), fixation on a line oscillating at higher frequencies should maintain the eye in a constant position. However, if the oscillations are of large amplitude, the "fixation" stimulus will be moving across the retina, which must reduce its efficacy as a position reference. Therefore, a movement FRF was measured under conditions similar to those of the previous experiments but with the reference line oscillating at $3.5 \mathrm{~Hz}$ with an amplitude of $10 \mathrm{~min}$.

Results

Figures 2 and 3 show the effects of removing the reference line on

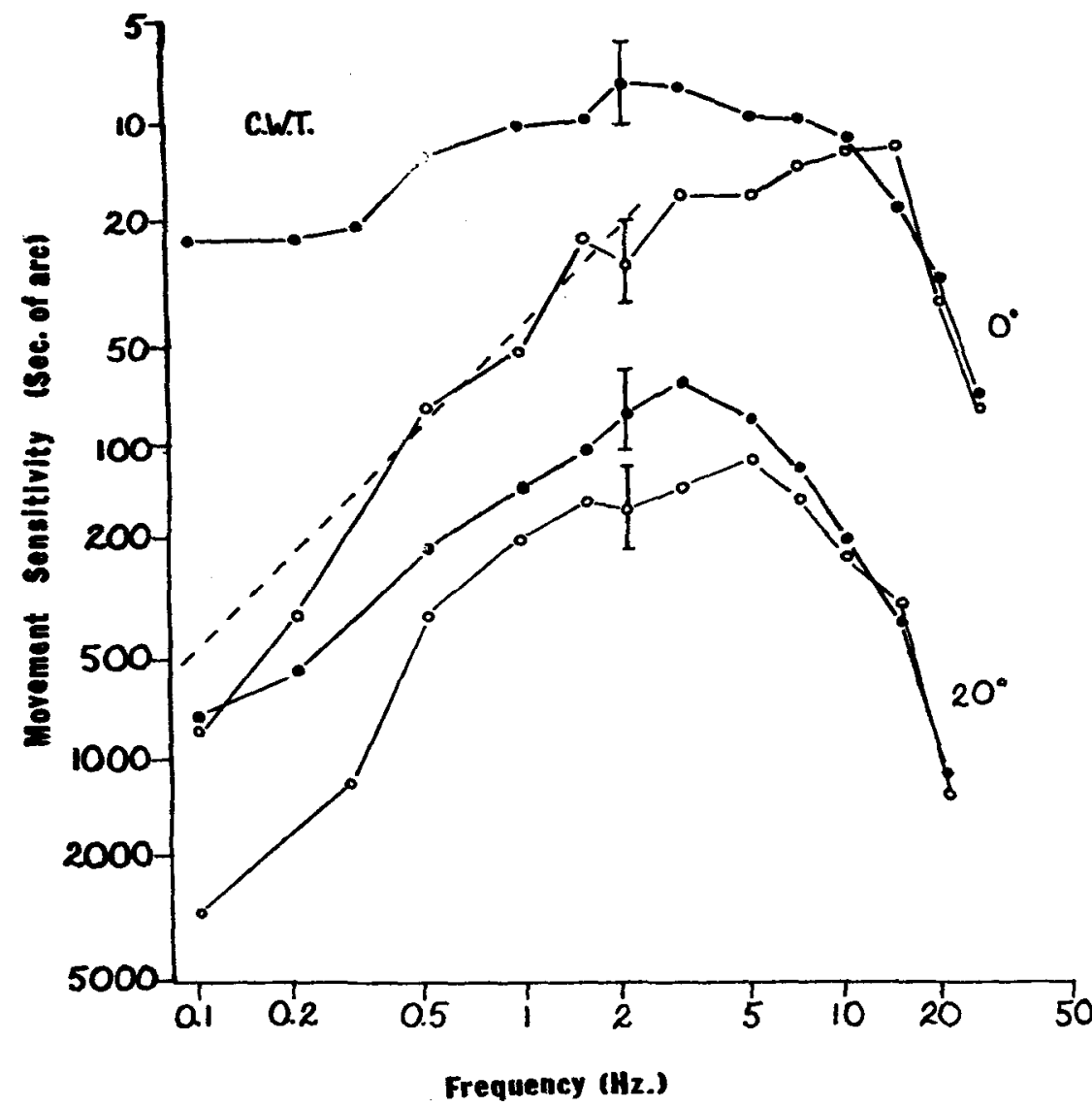

Fig. 3. As for Fig. 2, but with S C.W.T. Data with reference is replotted from Fig. 1.

movement sensitivity. For C.W.T., the data with reference line are replotted from Fig. 1. The data with reference for J.T. consist of new measurements which are similar to those in Fig. 1 within experimental error.

For both Ss, removal of the reference line has a marked effect on foveal movement sensitivity for frequencies below $10 \mathrm{~Hz}$, while it has a much smaller effect at $20 \mathrm{deg}$ eccentricity. For J.T. (Fig. 2), there is virtually no change in peripheral sensitivity after removal of the reference line. For C.W.T. (Fig. 3), there appears to be a small reduction in sensitivity, approximately $0.3 \mathrm{log}$ units.

The form of the foveal curves without a reference line resembles that of the peripheral curves, both with and without a reference line, The difference in sensitivity between foveal and peripheral curves without reference may therefore be described for both $\mathrm{Ss}$ as a reduction in sensitivity of about $0.8 \mathrm{log}$ unit (a factor of 6.3), together with a shift of the sensitivity curve along the frequency axis of about an octave (a factor of 2 ).

Role of Eye Movements

The role of tracking eye movements in the production of the present data is not regarded as significant, for the following reasons. Where the reference line was present, the $\mathbf{S}$ was instructed to base the judgments on relative displacement and not to track the target. With regard to Experiment II, the data reviewed by St. Cyr and Fender (1969) show that the gain of tracking eye movements is negligible above about $3 \mathrm{~Hz}$. Had tracking eye movements played a significant role, removal of the reference line should have had no effect on foveal sensitivity between 3 and $10 \mathrm{~Hz}$, since eye movement tracking is not possible on this frequency range. Similarly, the effect of tracking eye movements in the frequency region below $0.5 \mathrm{~Hz}$ can be ruled out, since the studies of sinusoidal movement tracking reviewed by St. Cyr and Fender show that the oculomotor gain and phase shift remain essentially constant below $0.5 \mathrm{~Hz}$, and hence can have no effect on the frequency dependence of the movement sensitivity.

In peripheral observations, there is no possibility that eye movements occurred, since a foveal fixation target was present.

Although these considerations rule out the effects of tracking eye 


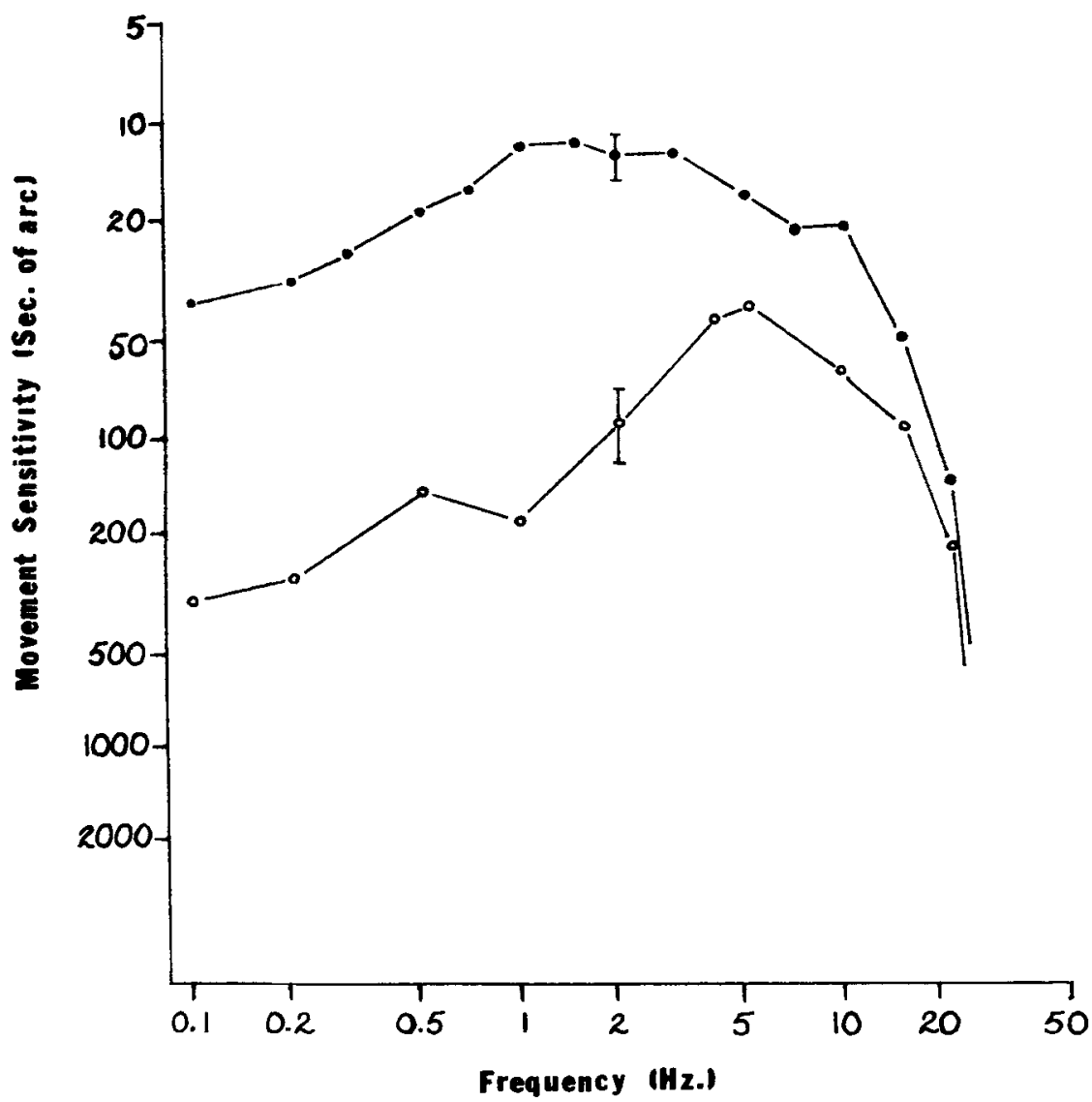

Fig. 4. Threshold sensitivity to sinusoidal movement with stationary reference (filled cirlces, replotted from Fig. 1) and with reference oscillating at $3.5 \mathrm{~Hz}$ (open circles). S C.W.T. Note similarity of effect to Fig. 3.

movements, it is possible that the "noise" introduced by involuntary movements might tend to reduce the sensitivity to retinal movement. This suggestion is supported by the data of Fender and Nye (1961), which show that the spectral density of involuntary eye movements during fixation remains approximately constant at $5 \mathrm{~min}$ of arc between 0.1 and $1 \mathrm{~Hz}$, then falls steadily to about $1 \mathrm{~min}$ of arc at $10 \mathrm{~Hz}$. Similarly, the difference between reference and no-reference conditions in the present experiment is reduced steadily between 1 and $10 \mathrm{~Hz}$. However, the effect of removing the reference does not remain constant between 0.1 and $1 \mathrm{~Hz}$ but increases by about $0.5 \mathrm{log}$ unit for both Ss. This observation indicates that the interference effect of involuntary eye movements could not be influencing the threshold in this low-frequency region, since the interference should remain constant between 0.1 and $1 \mathrm{~Hz}$.

If the no-reference FRF is not influenced by eye movements, how could fixation have been maintained in the absence of a reference line? We suggest that the background glow of the CRT could have served this function. It is possible that this diffuse light could act as a fixation target without providing position cues. Certainly it did not provide position cues; otherwise, there would not be a large difference between the reference and no-reference FRFs.

The subsidiary experiment provides further evidence that eye movements do not account for the effect of removal of the reference line. Movement sensitivity (open circles in Fig. 4) was measured for S C.W.T. with the reference oscillating at $3.5 \mathrm{~Hz}$, such as to provide an eye fixation cue but little retinal position information. Stationary reference sensitivity (full circles in Fig. 4) is shown for comparison. It has a similar form to that previously measured (Fig. 1).

With C.W.T., the FRF with the oscillating reference shows characteristics similar to the no-reference condition. Sensitivity in the high-frequency region is only slightly affected by oscillation of the reference, whereas sensitivity at low frequencies is reduced by about $1 \mathrm{log}$ unit. At very low frequencies, the oscillating reference curve (Fig. 4) falls less steeply than the no-reference curve (Fig. 3). This difference may well be attributable to the fact that the oscillating reference sensitivity is leveling off to about the same amplitude as the reference oscillations (10 $\mathrm{min})$. This suggests that oscillating the reference only reduces the position information when the oscillations are much larger than the threshold amplitude of the test line.

\section{DISCUSSION}

If the above reasoning is correct, the effects of eye movements are not important in producing the difference between reference and no-reference conditions below $1 \mathrm{~Hz}$. Consequently, the fact that removal or oscillation of the reference line affects foveal sensitivity below $10 \mathrm{~Hz}$ but not above suggests that the foveal movement sensitivity is mediated by a system consisting of at least two components. One component operates at the lower frequencies when there is a reference line, and the other component operates at the higher frequencies regardless of the presence of a reference line and is less sensitive than the first component below $10 \mathrm{~Hz}$. The second high-frequency component also characterizes the peripheral system, which appears to be insensitive to the presence or absence of a reference line. Leibowitz (1955) has previously suggested that the movement was detected by a position detector at long exposure durations and a velocity detector at short durations, but he did not consider the effects of eye movements.

The form of the sensitivity to sinusoidal movement can be used to determine the nature of two components. Consider first the high frequencies where sensitivity is limited very steeply and does not discriminate between the components. Here the target appears as a flickering bar of variable width. The sensitivity is therefore probably determined in a similar fashion to flicker sensitivity. Ives (1922), Veringa (1961), and Kelly (1969a, b, 1971) have suggested that high-frequency flicker sensitivity is limited by a retinal diffusion process that would probably operate in a similar manner with the high-frequency movement sensitivity even though, for the latter, the phase of the flicker varies continuously across the width of the perceived bar.

On the other hand, the low-frequency sensivitities do discriminate between the two components. If sensitivity in the foveal reference condition were determined by the retinal positions of the lines alone, it would be expected to be frequency independent, i.e., a 
horizontal line on the graph. The data in Figs. 1-4 show that, indeed, sensitivity is relatively unaffected by frequency up to $20 \mathrm{~Hz}$ in this condition. Furthermore, removal of the reference greatly reduces sensitivity. For these reasons, movement sensitivity in the foveal reference condition is attributed to a position detection mechanism.

Conversely, movement sensitivity in all three peripheral locations (Fig. 1) and in the no-reference conditions (Figs. 2 and 3 ) show strong frequency dependence. If movement sensitivity were determined by the maximum velocity occurring in the stimulus, sensitivity would increase in direct proportion to frequency (full line in Fig. 1 and dotted lines in Figs. 2 and 3 ). The data conform to this function between 0.1 and $1 \mathrm{~Hz}$, and hence support the hypothesis that the peripheral and no-reference sensitivity is determined by a velocity detection mechanism. Thus, the sinusoidal movement sensitivity data support Leibowitz's (1955) speculation that movement processing involves position detection in the fovea when a reference is available, and velocity detection in peripheral observation and when no reference is present.

On the basis of the straight line asymptotes to the low-frequency data, the velocity threshold in the fovea in the absence of a reference is calculated as about $3.3 \mathrm{~min}$ of arc/sec for J.T. and $3.0 \mathrm{~min}$ of $\mathrm{arc} / \mathrm{sec}$ for C.W.T. These values are slightly lower than classical measures of velocity sensitivity with no stationary reference stimuli (Aubert, 1886). Similarly, velocity threshold with a reference line for $\mathbf{S}$ J.T. was about $2.5,4.5$, and $10.0 \mathrm{~min}$ of $\operatorname{arc} / \mathrm{sec}$ at 5,10 , and $20 \mathrm{deg}$ eccentricity, respectively. Removal of the reference line hardly affected 20-deg sensitivity. For C.W.T., velocity threshold at $20 \mathrm{deg}$ eccentricity was about $7.0 \mathrm{~min}$ of arc/sec with a reference and about $12.0 \mathrm{~min}$ of arc/sec without. An interesting aspect of these data is that velocity threshold without a reference line is only reduced by about $0.5 \mathrm{log}$ unit from the fovea to $20 \mathrm{deg}$ eccentricity in both Ss, compared with twice this reduction in peak displacement sensitivity at $2-3 \mathrm{~Hz}$ oscillation. This reduction is also much less than the $0.9-\log$-unit reduction in photopic grating acuity from the fovea to 20 deg eccentricity found by Kerr (1971). Thus, the retinal periphery can be said to be specialized for velocity detection in that reduction in peripheral sensitivity for velocity is less by a factor of 2 than reduction in peripheral acuity relative to the fovea. The present data accord with those of Aubert (1886) in that under no conditions is the periphery more sensitive than the fovea, either to displacement or velocity.

Finally, it should be noted that, at maximum sensitivity, movement is detectable for only 8-10 sec of arc displacement. This is about $15 \%$ of the width of the bar, which is at the limit of optical resolution. Vernier acuity has about the same value for similar stimulus conditions (Tyler, 1972b), so that the temporal and spatial resolution of differences in luminance distributions are equivalent. It may be assumed that the line-spread function of a point of light has a normal distribution to a first approximation (Fry, 1955). A shift of $15 \%$ in the mean width of a normal distribution produces a redistribution of approximately $8 \%$ of the luminance of the bar from one side to the other, or a maximum contrast difference of $10 \%$. This is higher than the contrast threshold of about $1 \%$ obtained by Hecht and Mintz (1939). The difference may be due in part to the temporal integration that must take place for the movement to be observed. In any case, this discussion provides a conceptual framework in which discrimination of displacement of such fine magnitude can take place.

\section{REFERENCES}

AUBERT, H. Die Bewegungsempfindung. Archiv für die gesamte Physiologie, 1886 , 39, 347-370.

CLEMMESEN, V. Central and indirect vision of the light-adapted eye. Acta Physiologica Scandinavica, 1945, 9(Suppl. 27), 1-206.

de LANGE, H. Experiments on flicker and some calculations on an electrical analogue of the foveal systems. Physica, $1952,18,935-950$.

de LANGE, H. Research into the dynamic nature of the human fovea-cortex systems with intermittent and modulated light: $I$. Attenuation characteristics with white. and colored light. Journal of the Optical Society of America, 1958a, 48, 777-784.

de LANGE, H. Research into the dynamic nature of the human fovea-cortex systems with intermittent and modulated light: II. Phase shift in brightness and delay in color perception. Journal of the Optical Society of America, $1958 \mathrm{~b}, 48,784-789$.

FENDER, D. H., \& NYE, P. W. An investigation of the mechanisms of eye movement control. Kybernetik, 1961, 1 , $81-88$.

FRY, G. A. Blur of the retinal image. Columbus, Ohio: Ohio State University Press, 1955.

HECHT, S. \& MINTZ, E. U. The visibility of single lines at various illuminations and the retinal basis of visual resolution. Journal of General Physiology, 1939, 22, 593-612.

IVES, H. E. A theory of intermittent vision. Journal of the Optical Society of America. $1922,6,343-361$.
KEESEY, U. T. Variables determining flicker sensitivity in small fields. Journal of the Optical Society of America, 1970. $60,390-398$.

KELLY, D. H. Visual responses to time-dependent stimuli: I. Amplitude sensitivity measurements. Joumal of the Optical Society of America, 1961, 51. 422-429.

KELLY, D. H. Visual responses to time-iependent stimuli: IV. Effects of chromatic adaptation. Journal of the Optical Society of America, 1962, 52 , 940-947.

KELLY, D. H. Flickering patterns and lateral inhibition. Journal of the Optical Society of America, 1969a, 59 1361-1370.

KELLY, D. H. Diffusion model of linear flicker responses. Journal of the Optical Society of America, $1969 \mathrm{~b}, 59$, 1665-1670.

KELLY, D. H. Theory of flicker and transient responses: I. Uniform fields. Journal of the Optical Society of America, 1971, 61, 537-546.

KERR, J. L. Visual resolution in the periphery. Perception \& Psychophysics, $1971,9,377-378$

LEIBOWITZ, H. Effect of reference lines on the discrimination of movement. Joumal of the Optical Society of America, 1955 , 45, 829-830.

LEVINSON, J, Non-linear and spatial effects in the perception of ficker. Documenta Ophthalmologica, 1964, 18, 36-55.

McCOLGIN, F. H. Movement thresholds in peripheral vision. Journal of the Optical Society of America, 1960, 50, 774-779.

REGAN, D., \& TYLER, C. W. Temporal summation and its limit for wavelength changes: An analog of Bloch's law for color vision. Joumal of the Optical Society of America, 1971a, 61. 1414-1421.

REGAN, D., \& TYLER, c. W. Some dynamic features of colour vision. Vision Research, 1971b, 11, 43-5.6.

SCHICKMAN, G. M. Visual masking by low frequency sinusoidally modulated light. Journal of the Optical Society of America, 1970, 60, 107-117.

ST. CYR, G, J \& FENDER, D. $H$ Non-linearities of the human oculomotor system: Gain. Vision Research, 1969, 9 , 1235-1246.

TYLER, C. W, Stereoscopic depth movement: Two eyes less sensitive than one. Science, 1971, 174, 958-961.

TYLER, C. W. Temporal characteristics of apparent movement: Phi movement vs. Omega movement. Quarterly Journal of Experimental Psychology, 1972, in press.

TYLER, C. W. Periodic vernier acuity. Paper presented to the Association for Research in Vision and Opthalmology. April. $1972 b$.

van der TWEEL, $L$. Cortical responses to modulated light in the human subject. Acta Physiologica Scandinavica, 1960 , 48, 1-12.

VERINGA, F. Enige natuurkindige aspecten van het zien van gemoduleerd licht. Thesis, University of Amsterdam, 1961.

(Accepted for publication April 23, 1972.) NOTE

1. The term "frequency response function" (FRF) will be used in preference to the common "modulation transfer function" (MTF). MTF has the implication that the function is mathematically transformable to predict other types of response function, whereas FRF describes only the type of data that have been obtained. 